\title{
TOPOLOGICAL SEMIGROUPS AND CONTINUA WITH CUT POINTS ${ }^{1}$
}

\author{
W. M. FAUCETT
}

In this paper we are concerned with continua that possess cut points and that admit the structure of topological semigroups, herein called mobs. First, we discuss the relations between the existence of cut points in compact connected mobs and the algebraic structure of the mob. Next, we consider $D$-chains, as defined by Remage [6], in compact connected mobs. $D$-chains may be considered to be generalizations of the classical $A$-sets. In the final section, we center our attention on compact metric trees, or dendrites, that are mobs and show that under certain circumstances the algebraic structure of the non-cut points has a marked influence upon the algebraic structure of the entire mob.

The author wishes to acknowledge the advice and helpful suggestions of Professor A. D. Wallace in the preparation of this paper.

1. We define a mob to be a Hausdorff space together with a continuous associative multiplication. A submob in a mob $S$ is a nonvoid set $T$ contained in $S$ such that $T T \subset T$. A clan is a compact connected mob with unit. A left ideal of a mob $S$ is a nonvoid set $T, T \subset S$, such that $S T \subset T$. Right ideals and two-sided ideals are defined in the obvious manner. In this paper, we follow the terminology of Clifford [1] and shall reserve the letter $K$ to denote the minimal two-sided ideal and the letter $E$ to denote the set of idempotents. The symbol $\varnothing$ will designate the null set and the symbol * the Kuratowski closure operator.

The following lemma was stated and proved in [2] but we include it here for completeness.

Lemma 1.1. Let $S$ be a compact connected mob and let $t \in S \backslash K, K$ the minimal two-sided ideal of $S$. If $S \backslash t=A \cup B, A \mid B$, and if $K \subset A$, then $S t, t S$, and $S t S$ are contained in $A^{*}$.

Proof. Let $J$ be the union of all left ideals of $S$ contained in $A$. Since $K \subset A, J$ is non-null. As $J$ is open [4] and $S$ is connected, it follows that $t \in J^{*}$. Since $J^{*}$ is a left ideal, $S t \subset S J^{*} \subset J^{*} \subset A^{*}$. Similarly, $t S \subset A^{*}$ and $S t S \subset A^{*}$.

Presented to the Society, December 27, 1954 under the title Topological semigroups and trees; received by the editors December 17, 1954.

${ }^{1}$ This work was done under Contract N7-onr-434, Task Order III, Navy Department, Office of Naval Research. 
Let us define a two-sided ideal $T$ of a mob $S$ to be a prime ideal provided that whenever $S \backslash T$ is non-null then $S \backslash T$ is a submob.

THEOREM 1.1. Let $S$ be a clan and let $z$ be an element of $S$ such that $S \backslash z=A \cup B, A \mid B$. If $A$ is a two-sided ideal, then $A$ is a prime ideal if and only if $z \in E$.

Proof. If $A$ is a prime ideal, then $B^{*}$ is a submob and $z^{2} \in B^{*}$. The left ideal $A$ meets the right ideal $K$. Since $K$ is minimal, it follows that $K \subset A$. By Lemma $1.1, z^{2} \in A^{*}$. Thus $z^{2} \in A^{*} \cap B^{*}=z$. Hence $z \in E$.

Conversely, assume $z \in E$. For any $b \in B^{*}, S b$ is a connected set that meets $B^{*}$ and $A$. It follows that $z \in S b$; that is, $b^{\prime} b=z$ for some $b^{\prime} \in S$. Similarly, there exists $b^{\prime \prime} \in S$ such that $b b^{\prime \prime}=z$. Now suppose that for some $x, y \in B^{*}, x y \in A$. There exists $x^{\prime}$ and $y^{\prime \prime}$ such that $x^{\prime} x=z=y y^{\prime \prime}$. Thus $x^{\prime}(x y) y^{\prime \prime}=\left(x^{\prime} x\right)\left(y y^{\prime \prime}\right)=z^{2}=z \in S \backslash A$. Since $A$ is a two-sided ideal, this is a contradiction. This establishes that $B^{*}$ is a submob, which implies that $A$ is a prime ideal.

For any set $A$ contained in a mob $S$, let $L(A)=\varnothing$ if $A$ contains no left ideals of $S$. Otherwise, let $L(A)$ be the union of all left ideals of $S$ contained in $A$.

THEOREM 1.2. Let $S$ be a compact connected mob and let $e \in E \backslash K$. Further, let $C$ be the component of $S \backslash e$ containing $K$. If $L=L(S \backslash e)$, then $(S \backslash L) e \subset F(L)$. Moreover, if $C$ is a left ideal, then $(S \backslash L) e=e$.

Proof. Since $K \subset S \backslash e, L \neq \varnothing$. Let

$$
L^{\prime}=\{x \mid x e \in L\} \text {. }
$$

$L^{\prime}$ is a left ideal contained in $S \backslash e$. Thus $L^{\prime} \subset L$. Hence $x \in S \backslash L$ implies $x e \notin L$. But since $S$ is connected and since $L$ is open [4], it follows that $e \in L^{*}$. Therefore, if $x \in S \backslash L$, then $x e \in L^{*} \backslash L$. Since $L$ is open, $L^{*} \backslash L=F(L)$.

Now $S L \cup K$ is a connected set that is contained in $S \backslash e$ and meets $C$. Hence $S L \subset C$ and $S L^{*} \subset C^{*}$. Therefore, since $x \in S \backslash L$ implies $x e \notin L$ and $e$ is an element of $L^{*}$, we have $(S \backslash L) e \subset S L^{*} \backslash L \subset C^{*}$. If $C$ is a left ideal, then $C \subset L$. Hence $(S \backslash L) e \subset S L^{*} \backslash L \subset S L^{*} \backslash C \subset C^{*} \backslash C$. But since $S$ is connected, $e \in C^{*}$, and because $C$ is closed in $S \backslash e$, it follows that $C^{*} \backslash C=e$.

THeOREM 1.3. Let $S$ be a compact connected mob. If there exists a point $p \in S$ that cuts $K$, then either (i) $K=S p, K$ is a minimal left ideal, and every element of $K$ is a left zero for $S$; or (ii) $K=p S, K$ is a minimal right ideal, and every element of $K$ is a right zero for $S$. 
Proof. Ket $K \backslash p=A \cup B, A \mid B$. Clearly $p \in K$, since $K$ is connected. As $K$ is the union of disjoint groups, $p \in H(e)=e S e$, where $H(e)$ is the maximal subgroup containing $e$ for some $e \in E \cap K[1]$.

We claim $p=H(e)$. Suppose $H(e) \backslash p \neq \varnothing$. Since $H(e)$ is a compact connected topological group, $H(e) \backslash p$ is connected. Therefore, $H(e) \backslash p$ $\subset A$, or $H(e) \backslash p \subset B$. Assume $H(e) \backslash p \subset A$. For all $b \in B, b S b$ is a compact connected topological group that meets $B$. Since the maximal subgroups are disjoint, $b S b$ must be disjoint from $p S p$, as $p S p \subset A^{*}$. Therefore, we have $b S b \subset K \backslash p=A \cup B$. Since $b S b$ is connected and meets $B, b S b \subset B$ for all $b \in B$.

As we have assumed $H(e)$ to be nontrivial, there exists $y \in S$ such that $p y p \neq p$. Therefore, $p y p \in H(e) \backslash p \subset A$. Since $A \subset S \backslash B^{*}$, there exists an open set $U$ such that $p y p \in U$ and $U \cap B=\varnothing$. By the continuity of multiplication, there exist open sets $V$ and $V^{\prime}, p \in V$ and $p \in V^{\prime}$, such that $V y V^{\prime} \subset U$. Let $W=V \cap V^{\prime}$. But since $p \in B^{*}$, $W \cap B \neq \varnothing$. Thus $b y b \in U$ for some $b \in W \cap B$. But this contradicts the fact that $b S b \subset B$ for all $b \in B$.

We have established that $H(e)=p$. Since the maximal subgroups of $K$ are topologically equivalent [5], it follows that every maximal subgroup consists of a single element and that every element of $K$ is an idempotent.

For any $k \in K, S k(k S)$ is a minimal left (right) ideal and $S k \cap k S$ is a group containing $k$ [1]. Since in our situation every element of $K$ is idempotent, this intersection must be the single element $k$.

Fix $b^{\prime} \in B$. We assert that either $b^{\prime} S \subset B$ or $S b^{\prime} \subset B$. Suppose both $b^{\prime} S$ and $S b^{\prime}$ are not contained in $B$. Since $b^{\prime} \in b^{\prime} S$ and $b^{\prime} \in S b^{\prime}$, these two sets are not contained in $A$. Consequently, $p \in b^{\prime} S$ and $p \in S b^{\prime}$. But this is a contradiction since $b^{\prime} S \cap S b^{\prime}=b^{\prime} \neq p$. Thus either $b^{\prime} S \subset B$ or $S b^{\prime} \subset B$. Say $b^{\prime} S \subset B$.

For any $a \in A^{*}$, let us consider the minimal left ideal $S a$. Since $a \in S a, S a \nsubseteq B$. But since every left ideal meets every right ideal and since $b^{\prime} S \subset B, S a \nsubseteq A$. Therefore, $p \in S a$ for all $a \in A^{*}$.

We shall now show that $p \in S b$ for all $b \in B$. First, let $a^{\prime}$ be an arbitrary element of $A$. We claim $a^{\prime} S \subset A$. Suppose $a^{\prime} S \nsubseteq A$. Since $a^{\prime} \in a^{\prime} S$, it follows that $p \in a^{\prime} S$. But we have just shown that $p \in S a^{\prime}$. Consequently, we have $p \in a^{\prime} S \cap S a^{\prime}$. But this is a contradiction since $a^{\prime} S \cap S a^{\prime}=a^{\prime}$. This establishes our assertion that $a^{\prime} S \subset A$. For any $b \in B$, let us consider the minimal left ideal $S b$. Since $b \in S b, S b \nsubseteq A$. But since every left ideal meets every right ideal and since $S a^{\prime} \subset A$, $S b \nsubseteq B$. Consequently $p \in S b$ for all $b \in B$.

We have now shown $p \in S k, k \in K=A * \cup B$. Hence $S p \subset S k$ and since $S k$ is minimal, $S p=S k$ for each $k$. Thus $S p=S K$. But $K \subset K K$ 
$C S K \subset K$, which implies $K=S p$, a minimal left ideal.

It remains to prove that every element of $K$ is a left zero for $S$. Let $k^{\prime}$ be an arbitrary element of $K$. As we have already shown, $k^{\prime} S \cap S k^{\prime}=k^{\prime}$. But $S k^{\prime}$ is a left ideal contained in $K$. Since $K$ is a minimal left ideal, $S k^{\prime}=K$. As $k^{\prime} S \subset K=S k^{\prime}$, we have $k^{\prime} S \cap S k^{\prime}=k^{\prime} S$ $=k^{\prime}$. Thus $k^{\prime}$ is a left zero for $S$.

We obtain the dual conclusion if we assume $S b^{\prime} \subset B$.

The existence of maximal proper ideals in compact mobs has been discussed by Koch and Wallace [4]. In the next theorem we are concerned with the complement of a maximal proper two-sided ideal in a compact connected mob. We raise the query if the condition $S^{2}=S$ might not be replaced.

TheOREM 1.4. Let $S$ be a compact connected mob such that $S^{2}=S$. If $J$ is a maximal proper two-sided ideal of $S$, then for any closed set $M \subset A=S \backslash J$, the set $S \backslash M$ is connected.

Proof. Suppose $S \backslash M=P \cup Q, P \mid Q$. Since $K$ is connected and since $K \subset J=S-A \subset S \backslash M$, we can assume $K \subset P$. Let $T$ be the maximal two-sided ideal of $S$ contained in $P$. Since $T$ is open [4] and since $S$ is connected, it follows that $T^{*} \cap M \neq \varnothing$. For $m \in T^{*} \cap M$, we have $S m S \subset T^{*}$. Now, $T \subset P \subset P \cup M$. Certainly, $T^{*} \subset P^{*} \subset(P \cup M)^{*}$ $=P \cup M$. Therefore $S m S \subset T^{*} \subset P \cup M$. We claim $S A S \subset T^{*}$. If $A=m$, our assertion is obvious. On the other hand, if $A$ is nontrivial, it is known that $A \subset S m S$ [3]. Consequently, $S A S \subset S m S \subset T^{*} \subset P \cup M$.

By assumption, $Q \neq \varnothing$. Since $S^{2}=S, S=S E S$ [4]. Thus for any $q \in Q, q \in S e S$ for some $e \in E$. We claim $e \in J$. If $e \in A$, then $\operatorname{SeS} \subset S A S$ $\subset P \cup M=S \backslash Q$. SeS meets $P$ since $K \subset P$ and $S e S$ meets $Q$ since $q \in S e S$. Therefore, the connected set $S e S$ meets $M$. But this is a contradiction since $e \in J$ which implies that $S e S \subset J=S \backslash A \subset S \backslash M$.

2. For any set $N$ contained in a connected space $S$, let $\sigma(N)$ $=(S \backslash N)^{*}$. A closed set $N$ contained in $S$ is a nodal set if (i) $\sigma(N) \neq S$ and (ii) $N \cap \sigma(N)$ is at most a single point. If $N$ is a nodal set and if $N \cap \sigma(N)=z$, we shall say there exists a nodal decomposition at $z$. We shall use the notation $\delta(z): S=N \cup(S \backslash N)^{*}$ to denote a nodal decomposition at the point $z$. It is clear that $z$ is a cut point of $S$. Conversely, if $z$ is a cut point of $S$, then there exists a nodal decomposition at $z$.

For any non-null set $A$ contained in $S$, we define $D(A)$ to be the intersection of all the nodal sets containing $A$. A $D$-chain is a set $A, A \neq \varnothing$, such that $D(A)=A$. If $S$ is a continuum, then a $D$-chain is a sub-continuum [6].

We shall now state without proof a result due to Remage [6]. 
Lemma 2.1. Let $S$ be a continuum. Let $C$ be a $D$-chain and let $U$ be an open set containing $C$. If $x \in S \backslash U$, then there exists a nodal decomposition $\delta(z): S=N \cup(S \backslash N) *$ such that $z \in U, C \subset N$, and $x \in N$.

The next lemma is an immediate consequence of the above result.

Lemma 2.2. Let $S$ be a continuum, $M$ a sub-continuum of $S$, and $C$ a $D$-chain contained in $S \backslash M$. If $P=\{p: p \in S$ such that there exists $\left.\delta(p): S=N \cup(S \backslash N)^{*}, C \subset N, M \subset S \backslash N\right\}$, then $P^{*} \cap C \neq \varnothing$.

Lemma 2.3. Let $S$ be a compact connected mob such that there exists a nodal decomposition $\delta(p): S=N \cup(S \backslash N)^{*}$, with $K \subset S \backslash N$. If $A$ is a subset of $N$ such that for every $a \in A$ there exists $e \in E \cap N$ with $a \in S e$ $(a \in e S)$ and $e \in S a(e \in a S)$, then $A p=p(p A=p)$.

Proof. Suppose there exists $a \in A$ such that $a p \neq p$. Since $K \subset S \backslash N$, by Lemma 1.1 we have $a p \in S \backslash N$. By hypothesis, there exists $e \in E \cap N$ such that $a \in S e$. This implies that $a e=a$. Let $D^{\prime}=e S \cap N$. Now $e S$ is connected and is not contained in $S \backslash N$ since $e \in e S$ and $e \in N$. But $e S$ is not contained in $N$ since $K \subset S \backslash N$ and $e S$ meets $K$. Therefore, $p \in e S$ and $p \in D^{\prime}$. Now, $D^{\prime}$ is a continuum, being the intersection of a continuum and a nodal set. Since $a p \in a D^{\prime} \cap(S \backslash N)$ and since $a e=a \in a D^{\prime} \cap N, p$ is an element of $a D^{\prime}$; that is, $p=a d^{\prime}$ for some $d^{\prime} \in D^{\prime}$. By hypothesis $e \in S a$, which implies $e=s a$ for some $s \in S$. Now, $s p=s a d^{\prime} \in S p$, which is contained in $(S \backslash N)^{*}$ by Lemma 1.1. But $s p=s a d^{\prime}=(s a) d^{\prime}=e d^{\prime}$ and $e d^{\prime}=d^{\prime}$ since $d^{\prime} \in e S$ and $e$ is a left unit on $e S$. But this is a contradiction as $d^{\prime} \in N \backslash p=S \backslash(S \backslash N)^{*}$.

By duality $p A=p$.

THEOREM 2.1. Let $S$ be a compact connected mob and let $C$ be a $D$ chain contained in $S \backslash K$. If $C$ is a submob, then there exists $x \in C$ such that $x C=x=C x$.

Proof. Let

$$
\begin{gathered}
P=\left\{p: p \in S \text { such that there exists } \delta(p): S=N \cup(S \backslash N)^{*},\right. \\
C \subset N, K \subset S \backslash N\} .
\end{gathered}
$$

Let $K^{\prime}$ be the kernel of the compact connected submob $C$. For $e \in E \cap K^{\prime}, H=e C e$ is a compact connected topological group. $H$ clearly satisfies the conditions required of the set $A$ in Lemma 2.3. Therefore for any $p \in P, H p=p=p H$. Since $K^{\prime}=\bigcup\left\{e C e: e \in E \cap K^{\prime}\right\}$, it follows that $K^{\prime} p=p=p K^{\prime}$.

Now $T=\left\{x: K^{\prime} x=x=x K^{\prime}\right\}$ is closed. As $P \subset T$, we have $P^{*} \subset T^{*}$ $=T$. But by Lemma $2.2, P^{*} \cap C \neq \varnothing$, which implies that there exists $x \in C$ such that $K^{\prime} x=x=x K^{\prime}$. 
Because $C x=C\left(K^{\prime} x\right)=\left(C K^{\prime}\right) x=K^{\prime} x=x$ and dually, the conclusion is established.

TheOREM 2.2. If $S$ is a compact connected mob and if $A$ is a nontrivial $D$-chain, then $S \backslash A$ is not a maximal proper two-sided ideal.

Proof. Suppose $S \backslash A=J$ is a maximal proper two-sided ideal. Since $J$ is an ideal, $K \subset S \backslash A$. Let

$$
\begin{gathered}
P=\left\{p: p \in S \text { such that there exists } \delta(p): S=N \cup(S \backslash N)^{*},\right. \\
A \subset N, K \subset S \backslash N\} .
\end{gathered}
$$

Let $a$ be an arbitrary element of $A$. Since $A$ is nontrivial, it is known that there exists $e \in E \cap A$ such that $a \in S e(a \in e S)$ and $e \in S a(e \in a S)$ [3]. Hence $A$ satisfies the hypotheses of Lemma 2.3. Thus $A p=p$ $=p A$ for all $p \in P$.

Now since the set $T=\{x: A x=x=x A\}$ is closed and $P \subset T$, we have $P^{*} \subset T^{*}=T$. By Lemma 2.2, $P^{*} \cap A \neq \varnothing$, which implies that there exists $x \in A$ such that $A x=x=x A$. Therefore, since $J \cup x$ is a two-sided ideal containing $J$ properly, we have $J \cup x=S$. But this is a contradiction as $A$ is nontrivial by hypotheses.

3. We define a tree to be a nontrivial continuum such that every two points are separated by a third point. All the results in this section have been established for the more general compact Hausdorff case, but we shall consider only metric trees. We do this in order to simplify the presentation by invoking the results in the literature on metric trees.

The following results can be found in [8], or can be obtained by trivial arguments. Any two distinct points in a metric tree are joined by a unique simple arc. We shall denote the simple arc joining $a$ and $b$ in a metric tree $S$ by $(a, b)$. The arc $(a, b)$ is irreducibly connected between $a$ and $b$ and every point of the arc different from $a$ and $b$ separates $a$ and $b$ in $S$. If $M$ is a continuum contained in a metric tree $S$ and if $x \in S \backslash M$, then there exists $m^{\prime} \in M$ such that $\left(m^{\prime}, x\right) \cap M$ $=m^{\prime}$. Also every point of $S$ is either a cut point or an endpoint.

Lemma 3.1. Let $S$ be a mob. If $S$ is a metric tree, then either every element of $K$ is a left zero, or every element of $K$ is a right zero.

Proof. If $K$ is a single element, the conclusion is trivial. Otherwise, there exists a point in $S$ that cuts $K$ and the conclusion follows from Theorem 1.3.

Lемма 3.2. Let $S$ be a metric tree. Further, let $S$ be a mob and 
$e \in E \backslash K$. If $k^{\prime} \in K$ such that $\left(k^{\prime}, e\right) \cap K=k^{\prime}$, then $\left(k^{\prime}, e\right)$ is a compact abelian submob with e as a unit.

Proof. Since every point $t \in\left(k^{\prime}, e\right)$ different from $k^{\prime}$ and $e$ separates $k^{\prime}$ and $e$ in $S$, we have $S \backslash t=A \cup B, A \mid B$, with $k^{\prime} \in A$ and $e \in B$. As $K$ is a connected set contained in $S \backslash t$ and $K$ meets $A, K$ is contained in $A$. Now $e S$ is a connected set meeting both $A$ and $B$; hence $t \in e S$. Since $e S$ is closed, it follows that $\left(k^{\prime} e\right) \subset e S$. Dually for $S e$. Therefore, $e$ is a two-sided unit on $\left(k^{\prime}, e\right)$. Furthermore, $\left(k^{\prime}, e\right)$ is irreducibly connected between $k^{\prime}$ and $e$ and we can define a linear order in $\left(k^{\prime}, e\right)$ such that $k^{\prime} \leqq d$ for all $d \in\left(k^{\prime}, e\right)$ [9]. We assert that if $d^{\prime} \leqq d$ then $d^{\prime} \in d S$ and $d^{\prime} \in S d$. This follows from the fact that $d^{\prime}$ separates $d$ and $K$ and $d S$ and $S d$ are connected sets containing $d$ and meeting $K$.

By Lemma 3.1, we may assume every element of $K$ to be a left zero or a right zero. Say every element of $K$ is a left zero. Suppose there exist $x$ and $y, x, y \in\left(k^{\prime}, e\right)$, such that $x y \notin\left(k^{\prime}, e\right)$. An easy argument shows there exists $z \in S$ such that $S \backslash z=A \cup B, A \mid B, x y \in A$ and $\left(k^{\prime}, e\right) \subset B$. As $\left(k^{\prime}, e\right)$ can be linearly ordered, we define

$$
T=\left\{t: t \in\left(k^{\prime}, e\right), y \leqq t \leqq e\right\} .
$$

$T$ is connected [9]. Since $x y \in x T$ and $x \in x T$, it follows that $z \in x T$; that is, $z=x t^{\prime}$, for some $t^{\prime} \in T$. Since $y<t^{\prime}, y \in t^{\prime} S$. Thus $y=t^{\prime} s^{\prime}, s^{\prime} \in S$. Now if $z \notin K, K \subset S \backslash z$. Since $k^{\prime} \in B$ and since $K$ is connected, $K \subset B$. We have $z s^{\prime}=x t^{\prime} s^{\prime}=x y \in A$. But this is a contradiction since by Lemma $1.1, z s^{\prime} \in B^{*}=S \backslash A$. On the other hand, if $z \in K$, we have $z s^{\prime}=x t^{\prime} s^{\prime}=x y \neq z$. But again, this is a contradiction since we have assumed every element of $K$ to be a left zero.

We have now shown $\left(k^{\prime}, e\right)$ to be a compact submob. Indeed, $\left(k^{\prime}, e\right)$ is a compact submob irreducibly connected between two idempotents, one of which is a unit on $\left(k^{\prime}, e\right)$. The other is a zero on $\left(k^{\prime}, e\right)$, in view of the following consideration. We already know that $k^{\prime}$ is a left zero. But $\left(k^{\prime}, e\right) k^{\prime} \subset\left(k^{\prime}, e\right) \cap K=k^{\prime}$. Therefore $k^{\prime}$ is both a left and right zero on $\left(k^{\prime}, e\right)$. As shown in $[2],\left(k^{\prime}, e\right)$ is abelian.

If we assume every element in $K$ to be a right zero, we obtain the conclusion by duality.

Lemma 3.3. Let $S$ be a mob and let $k^{\prime}$ be a left (right) zero for $S$. Let $N$ be the set of endpoints of $S$ and let $e$ be a unit for $N, e \in N \cap E$. If $S$ is a metric tree, then $S=G N(N G)$ where $G=\left(k^{\prime}, e\right)$.

Proof. Clearly $N \subset G N$ since $e \in G$ and $e$ is a unit for $N$. Also $k^{\prime} \in G N$ as $k^{\prime}$ is a left zero. Let $x$ be a cut point, $x \neq k^{\prime}$. Thus $S \backslash x$ $=A \cup B, A \mid B$. Suppose $k^{\prime} \in B$. An easy argument shows $N \cap A^{*} \neq \varnothing$. 
Let $n \in N \cap A^{*}$. Obviously, $n \neq x$. $G n$ is a connected set meeting $A$ and $B$. Therefore $x \in G n$. This completes the proof since every element of $S$ is either a cut point or an endpoint.

THEOREM 3.1. Let $S$ be a metric tree, and let $N$ be the set of endpoints of $S$. If $S$ is a clan and if $N$ is contained in the center of $S$, then $S$ is an abelian clan with zero.

Proof. If $u$, the unit of $S$, is in $K$, then $S$ is a compact connected group and consequently does not contain a cut point. Therefore, $S$ cannot be a tree. Thus $u \notin K$. There exists $k^{\prime} \in K$ such that $\left(k^{\prime}, u\right) \cap K$ $=k^{\prime}$. By Lemma 3.2, $\left(k^{\prime}, u\right)$ is a compact abelian submob. Further, by Lemma 3.1, we may assume $k^{\prime}$ to be a left zero for $S$. By Lemma 3.3, $S=G N$ where $G=\left(k^{\prime}, u\right)$. Thus for any pair of elements $x$ and $x^{\prime}$ of $S$, we have $x=g n$ and $x^{\prime}=g^{\prime} n^{\prime}$ for some $g$ and $g^{\prime}$, elements of $G$, and for some $n$ and $n^{\prime}$, elements of $N$. Hence, $x x^{\prime}=g n g^{\prime} n^{\prime}=g g^{\prime} n n^{\prime}$ $=g^{\prime} g n^{\prime} n=g^{\prime} n^{\prime} g n=x^{\prime} x$. This establishes that $S$ is abelian. Since $S$ is abelian, the left zero $k^{\prime}$ must be a right zero also, hence unique.

THEOREM 3.2. Let $S$ be a metric tree and let $N$ be the set of endpoints of $S$. If $S$ is a mob and if $N$ is a group, then $S$ has a zero. Further, if $N$ is an abelian group, then $S$ is abelian.

Proof. Let $e$ be the identity element of $N$. Now, $e \notin K$. Otherwise, $e$ would be a left zero or a right zero by Lemma 3.1, contradicting the fact that $e$ is the identity element for the group $N$. There exists $k^{\prime} \in K$ such that $\left(k^{\prime}, e\right) \cap K=k^{\prime}$. By Lemma 3.3, $S=G N$, where $G=\left(k^{\prime}, e\right)$. If $K=k^{\prime}$, then certainly $k^{\prime}$ is a zero. Therefore, assume $k^{\prime \prime} \in K \backslash k^{\prime}$. Now $k^{\prime \prime}=g n$ for some $g \in G, g \neq k^{\prime}$, and for some $n \in N$. Since $N$ is a group, there exists $\theta(n) \in N$ such that $n \theta(n)=e$. Therefore, $k^{\prime \prime} \theta(n)=g n \theta(n)=g e$. Since $e$ is a unit on $G, g e=g \in S \backslash K$. But this is a contradiction since $k^{\prime \prime} \theta(n) \in K$. This establishes that $S$ has a zero.

It remains to be proved that if $N$ is an abelian group then $S$ is abelian. Since $k^{\prime}=0$, we now let $G=(0, e)$. By Lemma 3.3, $S=G N=N G$. From this it follows that multiplication by elements of $N$ is a homeomorphism on $S$. By Lemma $3.2, G=(0, e)$ is an abelian submob. Therefore, to establish the conclusion it is sufficient to show that every element of $N$ commutes with every element of $G$.

Fix $n \in N$. $G n$ and $n G$ are continua containing 0 and $n$ and clearly must be the unique arc joining 0 and $n$. Suppose for some $g \in G$, $n g \neq g n$. Since both $n g$ and $g n$ are elements of the arc $(o, n)$, which can be linearly ordered, we may assume $n g<g n$. Let

$$
T=\{t: t \in(0, n), 0 \leqq t \leqq n g\} .
$$


$T$ is compact and is irreducibly connected between 0 and $n g$ [9]. Consider the connected set $\theta(n) T n$. The element 0 is contained in $\theta(n) T n$. Also $g n \in \theta(n) T n$ since $\theta(n) n g n=e g n=g n$. It follows immediately that $T$ is contained properly in $\theta n T n$. But this is a contradiction to the known result that states that if $T$ is compact and if $T \subset a T b$ then $T=a T b[7]$.

Corollary 3.2.1. If $S$ is a compact metric mob irreducibly connected between two points that form a group, then $S$ is abelian.

\section{REFERENCES}

1. A. H. Clifford, Semigroups containing minimal ideals, Amer. J. Math. vol. 70 (1948) pp. 521-526.

2. W. M. Faucett, Compact semigroups irreducibly connected between two idempotents, Proc. Amer. Math. Soc. vol. 6 (1955) pp. 741-747.

3. W. M. Faucett, R. J. Koch and K. Numakura, Complements of maximal ideals in compact semigroups, to appear in Duke Math. J.

4. R. J. Koch and A. D. Wallace, Maximal ideals in compact semigroups, Duke Math. J. vol. 21 (1954) pp. 681-685.

5. K. Numakura, On bicompact semigroups, Math. J. of Okayama Univ. vol. 1 (1952) pp. 98-108.

6. R. Remage, Jr., Invariance and periodicity properties of nonalternating in the large transformations, Dissertation, University of Pennsylvania, 1950 (unpublished).

7. A. D. Wallace, Inverses in Euclidean mobs, Math. J. of Okayama Univ. vol. 3 (1953) pp. 23-28.

8. G. T. Whyburn, Analytic topology, Amer. Math. Soc. Colloquium Publications, vol. $28,1942$.

9. R. L. Wilder, Topology of Manifolds, Amer. Math. Soc. Colloquium Publications, vol.. $32,1949$.

TUlane University of Louisiana 